\title{
SCCA-IgM as a Potential Biomarker of Non-Alcoholic Fatty Liver Disease in Patients with Obesity, Prediabetes and Diabetes Undergoing Sleeve Gastrectomy
}

\author{
Silvia Bettini ${ }^{a, b}$ Emanuel Bordigato ${ }^{a, b} \quad$ Gabriella Milan ${ }^{a, b} \quad$ Chiara Dal Pra'a, b \\ Francesca Favaretto ${ }^{a, b}$ Anna Belligolia, ${ }^{a}$ Marta Sanna $a^{a, b}$ Roberto Serra ${ }^{a, b}$ \\ Mirto Foletto ${ }^{a, b}$ Luca Prevedello ${ }^{a, b}$ Luca Busetto ${ }^{a, b}$ Giorgio Fassina ${ }^{c}$ \\ Roberto Vettor ${ }^{a, b}$ Roberto Fabris ${ }^{a, b}$ \\ ${ }^{a}$ Center for the Study and Integrated Treatment of Obesity, University Hospital of Padua, \\ Padua, Italy; 'Internal Medicine 3, Department of Medicine, University Hospital of Padua, \\ Padua, Italy; 'Xeptagen S.p.A., Venice, Italy
}

\section{Keywords}

Obesity · Non-alcoholic fatty liver disease · SCCA-IgM · Prediabetes · Diabetes - Bariatric surgery

\begin{abstract}
Background: Non-alcoholic fatty liver disease (NAFLD) has a high prevalence in obesity and its presence should be screened. Laparoscopic sleeve gastrectomy (LSG) is an effective treatment for obesity, but its effects on NAFLD are still to be firmly established. The diagnosis of non-alcoholic steatohepatitis (NASH) is currently performed by liver biopsy, a costly and invasive procedure. Squamous cell carcinoma antigen-IgM (SCCA-IgM) is a biomarker of viral hepatitis to hepatocellular carcinoma development and its role in NAFLD to NASH progression has not yet been investigated. Objective: The aim of this study was to evaluate SCCA-IgM as a non-invasive biomarker of NAFLD/NASH in patients with different degrees of metaboliccomplicated obesity before and after LSG. Method: Fifty-six patients with obesity were studied before and 12 months after LSG; anthropometric, biochemical, clinical, and imaging data were collected. Results: At baseline steatosis was strongly associated with the glycaemic profile $(p=0.016)$ and was already present in prediabetic patients with obesity (82\%). Only 3 patients had an SCCA-IgM level above the normal cut-off. SCCA-IgM titre did not change
\end{abstract}


according to glycaemic profile or steatosis. Metabolic and inflammatory factors and transaminases significantly reduced after LSG-induced weight loss, except for SCCA-IgM. The ALT/ AST ratio decreased post-LSG correlated with BMI $(r=0.297, p=0.031)$, insulin $(r=0.354, p=$ $0.014)$, and triglycerides ( $r=0.355, p=0.009)$ reduction. Conclusions: Our results confirm the tight link between NAFLD and metabolic complications, suggesting prediabetes as a new risk factor of steatosis. SCCA-IgM does not seem to have a role in the identification and prognosis of NAFLD.

(C) 2019 The Author(s)

Published by S. Karger AG, Basel

\section{Introduction}

Non-alcoholic fatty liver disease (NAFLD) is characterized by excessive hepatic fat accumulation, associated with insulin resistance (IR), and defined by the presence of steatosis in $>5 \%$ of hepatocytes. The diagnosis of NAFLD requires the exclusion of both secondary causes and a daily alcohol consumption $\geq 30 \mathrm{~g}$ for men and $\geq 20 \mathrm{~g}$ for women $[1,2]$. The term NAFLD includes two pathologically distinct conditions with different prognoses: non-alcoholic fatty liver (NAFL) and NASH non-alcoholic steatohepatitis (NASH). NASH covers a wide spectrum of disease severity, ranging from fibrosis, to cirrhosis, to hepatocellular carcinoma (HCC).

NAFLD currently represents the most common liver disease in Western countries, being found in $25-30 \%$ of the general population. The reported prevalence of NAFLD varies according to the population studied and to the diagnostic criteria considered [3]. NAFLD is closely associated with the metabolic syndrome and mostly occurs in patients with obesity (60-95\%), type 2 diabetes mellitus (T2DM; 28-55\%), and hyperlipidaemia (27-92\%) [4]. NAFLD, evaluated by biopsy, was found in up to $95 \%$ of patients with obesity undergoing bariatric surgery [5]. Fibrosis is the most important prognostic factor in NAFLD and is correlated with liver-related outcomes and mortality.

According to recent guidelines [1], patients with metabolic risk factors (i.e., IR, obesity, or metabolic syndrome) have to be referred to diagnostic procedures for NAFLD (recommendation level A1) by ultrasound scan (US) and liver enzymes assay. Nevertheless, liver biochemistries can be in the normal range in patients with NAFLD [6, 7], and US or transient elastography, even if potentially more sensitive, are not considered reliable tools. Many serum markers and scores of fibrosis, such as the NAFLD fibrosis score (NFS), fibrosis 4 calculator (FIB-4), enhanced liver fibrosis (ELF), and FibroTest ${ }^{\circledR}$ [8-10], have proven acceptable diagnostic accuracy. In particular, the ALT/AST ratio is an index of severe liver damage and it is related to the degree of fibrosis [11]. Nanji et al. [12] reported a significant correlation between the ALT/AST ratio and the degree of fatty infiltration of the liver. The identification of advanced fibrosis or cirrhosis is less accurate by current available biomarkers and needs to be confirmed by liver biopsy. Even if this procedure is essential for NASH diagnosis and able to discriminate between NAFL and NASH, it is costly and invasive, and thus not suitable for general population studies [1, 2, 5, 9]. For all these reasons there is an increasing need to develop non-invasive assessment to identify NAFLD in patients with increased metabolic risk and a worse prognosis.

We focused our attention on squamous cell carcinoma antigen (SCCA), which belongs to the clade B subset of the serpins family [13]. The two isoforms SCCA1 (SERPINB3) and SCCA2 (SERPINB4) are physiologically expressed in squamous epithelia, in endothelial cells of the veins, in the walls of arteries [14], and in peripheral blood mononuclear cells [15]. It has been reported that SERPINB3 induces TGF $\beta$ expression in chronic liver disease [16] and promotes fibrogenesis in experimental models [17]. Data indicate that SERPINB3 protects cellular from apoptotic death caused by different kinds of stimuli [18], inducing cell proliferation and 
increasing invasiveness potential. SCCA1 and SCCA2 are overexpressed in squamous cell carcinoma of the uterine cervix, lung, head and neck, rectal colon, and pancreatic and liver tumours. Recently, an ELISA assay has been developed to detect SCCA isoforms (SERPINB3 and SERPINB4) complexed with natural IgM in human serum [19]. Monitoring SCCA-IgM levels over time appears to be a useful approach to identify patients with viral chronic hepatitis at higher risk for cirrhosis development [20]. Significant reduction of SCCA-IgM, lasting up to 6 months of follow-up, was observed only in HCV-positive patients with a sustained response to standard therapy [21]. A significant increase of SCCA-IgM over time has been shown only in patients with early cirrhosis (histologically proven) who developed HCC within 4 years of follow-up [22]. While the role of serpin in viral chronic hepatitis is well established, its possible use in the diagnosis of NAFLD/NASH has so far not been investigated.

Bariatric surgery is the most efficient treatment for losing weight, and improving obesity and diabetes reduces liver fat and NASH progression. Prospective data showed an improvement in all NASH histological lesions 1 year after surgical intervention [23]. Liver stiffness measurement by transient elastography and some fibrosis scores were significantly improved 12 months after bariatric surgery [24]. Finally, few studies regarding the effects of different bariatric procedures on liver fat are available, in particular for laparoscopic sleeve gastrectomy (LSG) [24-27]. The aim of our study was to evaluate SCCA-IgM as a non-invasive biomarker of liver fat-induced damage, considering patients with different degrees of metabolic-complicated obesity before and after weight loss and metabolic improvement obtained by bariatric surgery intervention.

\section{Methods}

\section{Patients}

Fifty-six patients with severe obesity ( 40 female and 16 male) as candidates for bariatric surgery were studied before and 12 months after the procedure. The patients were enrolled at the Centre for the Study and Integrated Treatment of Obesity, Padua University Hospital, in the period between 2013 and 2015, with a BMI greater than 35 in the presence of comorbidities, or with a BMI greater than 40, according to criteria for bariatric surgery [28]. The patients underwent a multidisciplinary baseline evaluation according to a standard clinical protocol and a complete medical history was taken regarding eating, smoking and drinking habits, drug and medications, past and current medical conditions, and past surgical procedures. All patients received a 4-week very-low-calorie diet (VLCD) immediately before surgery. Baseline evaluation was performed within 6 months before surgery and before the beginning of the VLCD period. LSG is performed as the first choice procedure at our centre except for patients affected by severe gastro-oesophageal reflux disease. LSG was performed by the same surgical team. Briefly, the procedure involved stomach longitudinal resection starting $4-5 \mathrm{~cm}$ from the pylorus with the preservation of the gastric antrum; full details have already been published elsewhere [29].

After surgery, patients were followed up for nutritional and medical management. A complete clinical re-evaluation was scheduled in all patients after 12 months from the procedure.

The specific exclusion criteria were: infection with hepatotropic viruses, excessive alcohol consumption, active cancer, auto-antibodies indicative of autoimmune hepatitis, genetic hemochromatosis, a current infection, predictor signs for cirrhosis or HCC at the US, and use of hepatotoxic drugs. The Padua Ethical Committee for Clinical Research approved the study (2892P, 10/06/2013) and each patient gave their informed written consent.

\section{Anthropometric Measurements}

All anthropometric measurements were taken with subjects wearing only light clothes without shoes. Height was measured to the nearest $0.01 \mathrm{~m}$ using a wall-mounted stadiometer. 
Bettini et al.: SCCA-IgM in NAFLD, Obesity, Prediabetes, and Diabetes

Body weight was determined to the nearest $0.1 \mathrm{~kg}$ using a calibrated balance beam scale. BMI was calculated as weight $(\mathrm{kg})$ divided by the height squared $\left(\mathrm{m}^{2}\right)$.

\section{Ultrasound Scan}

All patients were referred for a US of the upper abdomen in order to detect the presence of steatosis [30]. Patients were fasted from midnight of the day scheduled for the scan and instructed to take charcoal tablets to allow a better visualization of the abdominal organs. Each US was performed by the same sonographer within 6 months before surgery and before the beginning of the VLCD period.

\section{Biochemical Measurements}

For each patient we measured their fasting plasma glucose (FPG), insulin, lipid profile (total, high-, and low-density lipoprotein [LDL] cholesterol, and triglycerides [TG]), platelets, alanine aminotransferase (ALT), aspartate aminotransferase (AST), gamma-glutamyl transferase (GGT), highly sensitive C-reactive protein (hs-CRP), interleukin-6 (IL-6), and leptin. All blood tests were performed after $8 \mathrm{~h}$ of fasting. Venous blood samples were collected in tubes coated with $68 \mathrm{IU}$ of lithium heparin, with EDTA 8\% or with acrylic gel/micronized silica in the morning (Becton Dickison, East Rutherford, NJ, USA). Samples were stored at $-20^{\circ} \mathrm{C}$ until analysis.

All biochemical blood analyses were performed with a standard diagnostic kit according to the WHO first international reference standard: glucose (Glucose HK Gen.3, Roche Diagnostic, USA), insulin, IL-6 (IMMULITE 2000 Immunoassay, Siemens Healthcare GmbH, Germany), hs-CRP (CardioPhase High-Sensitivity C-Reactive Protein, Siemens Healthcare), and leptin (Leptin-RIA-CT, Mediagnost, Germany). Full blood counts (platelets) were measured by flow cytometry (Sysmex Europe GmbH, Germany), serum lipids by spectrophotometer (Roche Diagnostic, USA), and ALT, AST, and GGT titres were assayed by enzymatic method with the addition of pyridoxal-5-phosphate in compliance with IFCC reference methods [31]. LDL cholesterol was calculated according to Friedewald [32].

The concentration of circulating SCCA-IgM was detected by an ELISA assay (Hepa-IC, provided by Xeptagen SpA, Venice, Italy), according to manufacturer's instructions [19]. The amount of total SCCA-IgM complex was expressed in arbitrary units per millilitre $(\mathrm{AU} / \mathrm{mL})$ and the value of $200 \mathrm{AU} / \mathrm{mL}$ was used as the cut-off [33].

The homeostasis model assessment (HOMA) was used to calculate the IR index (HOMA-IR) as: [fasting serum insulin $(\mu \mathrm{U} / \mathrm{mL}) \times \mathrm{FPG}(\mathrm{mmol} / \mathrm{L})] / 22.5$, as previous described [34]. A 3-h 75-g oral glucose tolerance test (OGTT) was performed by monitoring blood glucose and insulin plasma levels at basal time and 30, 90, 120, 150, and 180 min after glucose load ( $180 \mathrm{~mL}$ of syrup with $82.5 \mathrm{~g}$ of glucose monohydrate equal to $75 \mathrm{~g}$ of glucose) according to WHO standards [35]. The glucose and insulin area under the curve were calculated using the trapezoidal method. In patients affected by obesity and diabetes, OGTT was not performed and, if they were treated with insulin, fasting insulin was not measured and HOMA-IR was not calculated.

\section{Statistical Analysis}

Data were analysed using Statistical Package for the Social Sciences software (SPSS, v.23.0; IBM Corp., Armonk, NY, United States). All variables were tested for a normal distribution (Shapiro-Wilk test); comparisons with coupled data were carried out using the Wilcoxon signed-rank test, whereas the Mann-Whitney U test and Kruskal-Wallis test were employed for independent samples. The $\chi^{2}$ test was used to analyse the association between categorical variables. Spearman's correlation coefficient $(r)$ and $p$ values were calculated for statistical correlations between the analysed variables. In all analyses, a $p$ value $<0.05$ was considered to be statistically significant. 
Bettini et al.: SCCA-IgM in NAFLD, Obesity, Prediabetes, and Diabetes

Table 1. Anthropometric characteristics and biochemical parameters before and after surgery in patients with obesity divided into no steatosis and with steatosis subgroups according to the presence of steatosis assayed by liver US

\begin{tabular}{|c|c|c|c|c|c|}
\hline & \multicolumn{2}{|l|}{ No steatosis } & \multicolumn{2}{|l|}{ Steatosis } & \multirow{3}{*}{$\begin{array}{l}p \\
\text { before }\end{array}$} \\
\hline & \multicolumn{2}{|c|}{$n=16(3 \mathrm{M} / 13 \mathrm{~F})$} & \multicolumn{2}{|c|}{$n=40(13 \mathrm{M} / 27 \mathrm{~F})$} & \\
\hline & before & after & before & after & \\
\hline Age, years & $\begin{array}{l}50.5 \\
(40-57)\end{array}$ & - & $\begin{array}{l}47 \\
(40.3-56.8)\end{array}$ & - & 0.957 \\
\hline Weight, kg & $\begin{array}{l}122.5 \\
(105.6-159.3)\end{array}$ & $\begin{array}{l}89.0 \\
(72.6-116.3)^{* * *}\end{array}$ & $\begin{array}{l}127.3 \\
(112.0-149.0)\end{array}$ & $\begin{array}{l}92.5 \\
(75.2-103.3)^{* * *}\end{array}$ & 0.779 \\
\hline BMI, $\mathrm{kg} / \mathrm{m}^{2}$ & $\begin{array}{l}44.38 \\
(40.11-58.01)\end{array}$ & $\begin{array}{l}32.33 \\
(28.38-42.76)^{* * *}\end{array}$ & $\begin{array}{l}46.23 \\
(40.19-51.86)\end{array}$ & $\begin{array}{l}33.41 \\
(29.14-37.49)^{* * *}\end{array}$ & 0.800 \\
\hline FPG, mmol/L & $\begin{array}{l}5.5 \\
(5.1-5.9)\end{array}$ & $\begin{array}{l}4.6 \\
(4.4-4.9)^{* *}\end{array}$ & $\begin{array}{l}5.6 \\
(5.1-7.0)\end{array}$ & $\begin{array}{l}4.7 \\
(4.2-5.1)^{* * *}\end{array}$ & 0.490 \\
\hline Insulin, mIU/L & $\begin{array}{l}12 \\
(8-22)\end{array}$ & $(5-10)^{* *}$ & $\begin{array}{l}18 \\
(10-28)\end{array}$ & 7 & 0.097 \\
\hline HOMA-IR, $\mathrm{mIU} \cdot \mathrm{mmol} / \mathrm{L}^{2}$ & $\begin{array}{l}2.80 \\
(1.93-5.30)\end{array}$ & $\begin{array}{l}1.53 \\
(1.06-1.97)^{* *}\end{array}$ & $\begin{array}{l}5.33 \\
(2.50-8.30)\end{array}$ & $\begin{array}{l}1.46 \\
(0.74-2.20)^{* * *}\end{array}$ & 0.067 \\
\hline AST, U/L & $\begin{array}{l}21 \\
(17-24)\end{array}$ & $\begin{array}{l}19 \\
(14-21)\end{array}$ & $\begin{array}{l}24 \\
(17-34)\end{array}$ & $\begin{array}{l}18 \\
(14-21)^{* * *}\end{array}$ & 0.182 \\
\hline ALT, U/L & $\begin{array}{l}19 \\
(17-24)\end{array}$ & $\begin{array}{l}16 \\
(14-22)\end{array}$ & $\begin{array}{l}30 \\
(20-41)\end{array}$ & $\begin{array}{l}16 \\
(13-20)^{* * *}\end{array}$ & 0.019 \\
\hline ALT/AST & $\begin{array}{l}0.98 \\
(0.83-1.17)\end{array}$ & $\begin{array}{l}0.88 \\
(0.74-1.16)\end{array}$ & $\begin{array}{l}1.11 \\
(0.95-1.59)\end{array}$ & $\begin{array}{l}0.92 \\
(0.69-1.18)^{* * *}\end{array}$ & 0.046 \\
\hline GGT, U/L & $\begin{array}{l}14 \\
(11-24)\end{array}$ & $\begin{array}{l}14 \\
(8-17)\end{array}$ & $\begin{array}{l}23 \\
(18-45)\end{array}$ & $\begin{array}{l}13 \\
(9-23)^{* * *}\end{array}$ & 0.540 \\
\hline PLTS, $10^{9} / \mathrm{L}$ & $\begin{array}{l}241 \\
(207-334)\end{array}$ & $\begin{array}{l}212 \\
(183-275)^{* *}\end{array}$ & $\begin{array}{l}258 \\
(219-321)\end{array}$ & $\begin{array}{l}233 \\
(197-266)^{* * *}\end{array}$ & 0.942 \\
\hline T Chol, mg/dL & $\begin{array}{l}194 \\
(163-216)\end{array}$ & $\begin{array}{l}177 \\
(159-219)\end{array}$ & $\begin{array}{l}189 \\
(171-210)\end{array}$ & $\begin{array}{l}183 \\
(153-200)^{*}\end{array}$ & 0.935 \\
\hline $\mathrm{LDL}, \mathrm{mg} / \mathrm{dL}$ & $\begin{array}{l}112 \\
(94-153)\end{array}$ & $\begin{array}{l}102 \\
(81-128)^{*}\end{array}$ & $\begin{array}{l}123 \\
(104-138)\end{array}$ & $\begin{array}{l}111 \\
(84-123)^{* * *}\end{array}$ & 0.580 \\
\hline $\mathrm{HDL}, \mathrm{mg} / \mathrm{dL}$ & $\begin{array}{l}49 \\
(42-65)\end{array}$ & $\begin{array}{l}57 \\
(51-69)^{* * *}\end{array}$ & $\begin{array}{l}45 \\
(36-52)\end{array}$ & $\begin{array}{l}55 \\
(49-65)^{* * *}\end{array}$ & 0.084 \\
\hline $\mathrm{N}-\mathrm{HDL}, \mathrm{mg} / \mathrm{dL}$ & $\begin{array}{l}135 \\
(111-172)\end{array}$ & $\begin{array}{l}116 \\
(97-155)^{*}\end{array}$ & $\begin{array}{l}147 \\
(125-160)\end{array}$ & $\begin{array}{l}124 \\
(97-141)^{* * *}\end{array}$ & 0.474 \\
\hline $\mathrm{TG}, \mathrm{mg} / \mathrm{dL}$ & $\begin{array}{l}96 \\
(70-105)\end{array}$ & $\begin{array}{l}76 \\
(57-100)^{*}\end{array}$ & $\begin{array}{l}122 \\
(71-180)\end{array}$ & $\begin{array}{l}75 \\
(57-118)^{* * *}\end{array}$ & 0.125 \\
\hline hs-CRP, mg/L & $\begin{array}{l}5.22 \\
(1.88-8.50)\end{array}$ & $\begin{array}{l}2.24 \\
(0.79-4.62)^{*}\end{array}$ & $\begin{array}{l}5.46 \\
(3.18-7.83)\end{array}$ & $\begin{array}{l}1.16 \\
(0.63-3.54)^{* * *}\end{array}$ & 0.451 \\
\hline Leptin, $\mu \mathrm{g} / \mathrm{L}$ & $\begin{array}{l}40.5 \\
(26.8-65.0)\end{array}$ & $\begin{array}{l}16.0 \\
(12.0-22.0)^{*}\end{array}$ & $\begin{array}{l}37.2 \\
(26.4-50.3)\end{array}$ & $\begin{array}{l}12.9 \\
(7.0-20.8)^{* * *}\end{array}$ & 0.383 \\
\hline IL-6, ng/mL & $\begin{array}{l}2.6 \\
(1.9-5.2)\end{array}$ & $\begin{array}{l}1.9 \\
(1.9-2.2)^{* *}\end{array}$ & $\begin{array}{l}2.2 \\
(1.9-3.7)\end{array}$ & $\begin{array}{l}1.9 \\
(1.9-2.6)\end{array}$ & 0.294 \\
\hline SCCA-IgM, AU/L & $\begin{array}{l}59.76 \\
(28.19-92.18)\end{array}$ & $\begin{array}{l}48.15 \\
(28.01-81.12)\end{array}$ & $\begin{array}{l}55.23 \\
(32.18-88.19)\end{array}$ & $\begin{array}{l}52.31 \\
(33.55-88.76)\end{array}$ & 0.849 \\
\hline
\end{tabular}

Data are presented as the median value (25th-75th percentile). The differences in variable distributions among the groups before laparoscopic sleeve gastrectomy (LSG) were assayed with the Mann-Whitney U test. The differences in variable distributions within each group before versus after LSG were assayed with Wilcoxon signed-ranks for paired samples (non-normally distributed variables). ${ }^{*} p<0.05$; ${ }^{* *} p<0.01$; ${ }^{* *} p<0.001$. US, ultrasound scan; M, male; F, female; BMI, body mass index; FPG, fasting plasma glucose; HOMA-IR, homeostasis model assessment-insulin resistance index; AST, aspartate aminotransferase; ALT, alanine aminotransferase; GGT, gamma-glutamyl transferase; PLTS, platelets; T Chol, total cholesterol; LDL, low-density lipoprotein-cholesterol; HDL, high-density lipoprotein cholesterol; N-HDL, non-HDL-cholesterol; TG, triglycerides; hs-CRP, highsensitivity C-reactive protein; IL-6, interleukin-6; SCCA-IgM, squamous cell carcinoma antigen - immunoglobulin M complexes. 
Table 2. Anthropometric characteristics and biochemical parameters before and after surgery in patients with obesity divided according to glycaemic profile: with normoglycemia (ob N), prediabetes (ob preDM), and diabetes (ob T2DM)

\begin{tabular}{|c|c|c|c|c|c|c|c|}
\hline & \multirow{2}{*}{\multicolumn{2}{|c|}{$\begin{array}{l}\text { Normoglycaemia (ob N) } \\
n=22(5 \mathrm{M} / 17 \mathrm{~F})\end{array}$}} & \multirow{2}{*}{\multicolumn{2}{|c|}{$\frac{\text { Prediabetes (ob preDM) }}{n=17(6 \mathrm{M} / 11 \mathrm{~F})}$}} & \multirow{2}{*}{\multicolumn{2}{|c|}{$\begin{array}{l}\text { Diabetes (ob T2DM) } \\
n=17(5 \mathrm{M} / 12 \mathrm{~F})\end{array}$}} & \multirow{3}{*}{$\begin{array}{l}p \\
\text { before }\end{array}$} \\
\hline & & & & & & & \\
\hline & before & after & before & after & before & after & \\
\hline Age, years & $\begin{array}{l}41.5 \\
(36.8-54.5)\end{array}$ & - & $\begin{array}{l}54 \\
(42.5-60.5)\end{array}$ & - & $\begin{array}{l}51 \\
(41-56.5)\end{array}$ & - & 0.065 \\
\hline Weight, kg & $\begin{array}{l}126.0 \\
(108.0-161.1)\end{array}$ & $\begin{array}{l}89.0 \\
(76.2-111.9)^{* * *}\end{array}$ & $\begin{array}{l}132.0 \\
(110.5-151.5)\end{array}$ & $\begin{array}{l}100.7 \\
(74.3-109.3)^{* * *}\end{array}$ & $\begin{array}{l}127.0 \\
(101.0-144.0)\end{array}$ & $\begin{array}{l}86.0 \\
(72.3-99.0)^{* * *}\end{array}$ & 0.823 \\
\hline BMI, kg/m² & $\begin{array}{l}45.97 \\
(42.46-52.90)\end{array}$ & $\begin{array}{l}33.27 \\
(27.59-40.45)^{* * *}\end{array}$ & $\begin{array}{l}46.87 \\
(41.72-52.10)\end{array}$ & $\begin{array}{l}34.41 \\
(29.70-38.27)^{* * *}\end{array}$ & $\begin{array}{l}44.47 \\
(39.09-50.72)\end{array}$ & $\begin{array}{l}30.07 \\
(28.88-34.26)^{* * *}\end{array}$ & 0.489 \\
\hline $\mathrm{FPG}, \mathrm{mmol} / \mathrm{L}$ & $\begin{array}{l}5.2 \\
(5-5.4)\end{array}$ & $\begin{array}{l}4.6 \\
(4.3-4.7)^{* * *}\end{array}$ & $\begin{array}{l}5.8 \\
(5.3-6.1)\end{array}$ & $\begin{array}{l}4.6 \\
(4.5-4.9)^{* * *}\end{array}$ & $\begin{array}{l}7.3 \\
(6.1-8.6)\end{array}$ & $\begin{array}{l}5.1 \\
(4.1-5.4)^{* * *}\end{array}$ & $<0.001$ \\
\hline Insulin, mIU/L & $\begin{array}{l}13.5 \\
(9.8-22.3)\end{array}$ & $\begin{array}{l}7.0 \\
(4.5-10.0)^{* *}\end{array}$ & $\begin{array}{l}20.0 \\
(9.5-33)\end{array}$ & $\begin{array}{l}7.1 \\
(5.3-10.5)^{* * *}\end{array}$ & $\begin{array}{l}17.3 \\
(7.1-28.7)\end{array}$ & $\begin{array}{l}8.1 \\
(3.1-11.7)^{* *}\end{array}$ & 0.273 \\
\hline $\begin{array}{l}\text { HOMA-IR, } \\
\mathrm{mIU} \cdot \mathrm{mmol} / \mathrm{L}^{2}\end{array}$ & $\begin{array}{l}2.98 \\
(1.86-5.24)\end{array}$ & $\begin{array}{l}1.46 \\
(0.84-2.07)^{* * *}\end{array}$ & $\begin{array}{l}5.87 \\
(2.46-8.39)\end{array}$ & $\begin{array}{l}1.49 \\
(1.12-2.35)^{* * *}\end{array}$ & $\begin{array}{l}5.89 \\
(2.29-9.86)\end{array}$ & $\begin{array}{l}1.77 \\
(0.67-2.62)^{* *}\end{array}$ & 0.049 \\
\hline AST, U/L & $\begin{array}{l}20 \\
(16-25)\end{array}$ & $\begin{array}{l}16 \\
(14-19)^{* * *}\end{array}$ & $\begin{array}{l}24 \\
(20-34)\end{array}$ & $\begin{array}{l}20 \\
(15-23)^{* *}\end{array}$ & $\begin{array}{l}25 \\
(17-36)\end{array}$ & $\begin{array}{l}19 \\
(15-22)^{* *}\end{array}$ & 0.154 \\
\hline ALT, U/L & $\begin{array}{l}20 \\
(17-32)\end{array}$ & $\begin{array}{l}15 \\
(14-17)^{* * *}\end{array}$ & $\begin{array}{l}29 \\
(17-42)\end{array}$ & $\begin{array}{l}18 \\
(12-20)^{* *}\end{array}$ & $\begin{array}{l}24 \\
(22-40)\end{array}$ & $\begin{array}{l}19 \\
(14-23)^{* * *}\end{array}$ & 0.299 \\
\hline ALT/AST & $\begin{array}{l}1.06 \\
(0.88-1.45)\end{array}$ & $\begin{array}{l}0.88 \\
(0.73-1.19)^{*}\end{array}$ & $\begin{array}{l}1.00 \\
(0.81-1.36)\end{array}$ & $\begin{array}{l}0.90 \\
(0.79-1.00)\end{array}$ & $\begin{array}{l}1.24 \\
(0.96-1.42)\end{array}$ & $\begin{array}{l}0.93 \\
(0.69-1.23)^{*}\end{array}$ & 0.351 \\
\hline GGT, U/L & $\begin{array}{l}18 \\
(15-40)\end{array}$ & $\begin{array}{l}12 \\
(9-18)^{*}\end{array}$ & $\begin{array}{l}22 \\
(15-51)\end{array}$ & $\begin{array}{l}14 \\
(8-27)^{*}\end{array}$ & $\begin{array}{l}20 \\
(16-31)\end{array}$ & $\begin{array}{l}15 \\
(9-23)^{*}\end{array}$ & 0.815 \\
\hline PLTS, $10^{9} / \mathrm{L}$ & $\begin{array}{l}281 \\
(234-339)\end{array}$ & $\begin{array}{l}238 \\
(194-287)^{* * *}\end{array}$ & $\begin{array}{l}234 \\
(193-272)\end{array}$ & $\begin{array}{l}204 \\
(179-241)^{*}\end{array}$ & $\begin{array}{l}263 \\
(218-322)\end{array}$ & $\begin{array}{l}233 \\
(216-267)\end{array}$ & 0.130 \\
\hline T Chol, mg/dL & $\begin{array}{l}178 \\
(161-217)\end{array}$ & $\begin{array}{l}168 \\
(156-208)\end{array}$ & $\begin{array}{l}208 \\
(185-224)\end{array}$ & $\begin{array}{l}187 \\
(166-198)^{*}\end{array}$ & $\begin{array}{l}186 \\
(160-195)\end{array}$ & $\begin{array}{l}179 \\
(152-195)\end{array}$ & 0.078 \\
\hline LDL, mg/dL & $\begin{array}{l}112 \\
(95-143)\end{array}$ & $\begin{array}{l}101 \\
(83-127)^{* *}\end{array}$ & $\begin{array}{l}135 \\
(116-157)\end{array}$ & $\begin{array}{l}111 \\
(98-131)^{* * *}\end{array}$ & $\begin{array}{l}115 \\
(88-131)\end{array}$ & $\begin{array}{l}107 \\
(70-124)\end{array}$ & 0.104 \\
\hline $\mathrm{HDL}, \mathrm{mg} / \mathrm{dL}$ & $\begin{array}{l}49 \\
(38-55)\end{array}$ & $\begin{array}{l}56 \\
(49-70)^{* * *}\end{array}$ & $\begin{array}{l}48 \\
(45-53)\end{array}$ & $\begin{array}{l}58 \\
(50-64)^{*}\end{array}$ & $\begin{array}{l}43 \\
(34-51)\end{array}$ & $\begin{array}{l}53 \\
(50-68)^{* * *}\end{array}$ & 0.192 \\
\hline $\mathrm{N}-\mathrm{HDL}, \mathrm{mg} / \mathrm{dL}$ & $\begin{array}{l}129 \\
(114-177)\end{array}$ & $\begin{array}{l}112 \\
(97-152)^{* *}\end{array}$ & $\begin{array}{l}149 \\
(138-177)\end{array}$ & $\begin{array}{l}129 \\
(112-147)^{* * *}\end{array}$ & $\begin{array}{l}141 \\
(110-155)\end{array}$ & $\begin{array}{l}118 \\
(89-139)\end{array}$ & 0.207 \\
\hline $\mathrm{TG}, \mathrm{mg} / \mathrm{dL}$ & $\begin{array}{l}89 \\
(65-118)\end{array}$ & $\begin{array}{l}67 \\
(52-98)^{* * *}\end{array}$ & $\begin{array}{l}105 \\
(66-161)\end{array}$ & $\begin{array}{l}71 \\
(58-107)^{* *}\end{array}$ & $\begin{array}{l}148 \\
(102-183)\end{array}$ & $\begin{array}{l}87 \\
(68-130)^{* *}\end{array}$ & 0.036 \\
\hline hs-CRP, mg/L & $\begin{array}{l}5.45 \\
(2.88-8.39)\end{array}$ & $\begin{array}{l}2.30 \\
(1.03-5.11)^{*}\end{array}$ & $\begin{array}{l}4.95 \\
(2.41-7.86)\end{array}$ & $\begin{array}{l}1.09 \\
(0.75-5.17)^{*}\end{array}$ & $\begin{array}{l}6.64 \\
(2.48-8.84)\end{array}$ & $\begin{array}{l}1.02 \\
(0.38-2.73)^{* *}\end{array}$ & 0.809 \\
\hline Leptin, $\mu \mathrm{g} / \mathrm{L}$ & $\begin{array}{l}44.0 \\
(26.8-65.0)\end{array}$ & $\begin{array}{l}16.7 \\
(9.0-22.0)^{* * *}\end{array}$ & $\begin{array}{l}40.0 \\
(31.5-53.4)\end{array}$ & $\begin{array}{l}13.0 \\
(6.8-22.0)^{* * *}\end{array}$ & $\begin{array}{l}29.0 \\
(25.0-43.0)\end{array}$ & $\begin{array}{l}12.0 \\
(5.1-20.5)^{*}\end{array}$ & 0.130 \\
\hline IL-6, ng/mL & $\begin{array}{l}2.2 \\
(1.9-4.1)\end{array}$ & $\begin{array}{l}1.9 \\
(1.9-2.2)^{*}\end{array}$ & $\begin{array}{l}1.9 \\
(1.9-3.5)\end{array}$ & $\begin{array}{l}1.9 \\
(1.9-3.8)\end{array}$ & $\begin{array}{l}3.2 \\
(1.9-4.3)\end{array}$ & $\begin{array}{l}1.9 \\
(1.9-2.3)^{*}\end{array}$ & 0.360 \\
\hline SCCA-IgM, AU/L & $\begin{array}{l}67.50 \\
(32.18-91.92)\end{array}$ & $\begin{array}{l}52.35 \\
(38.57-81.08)\end{array}$ & $\begin{array}{l}52.62 \\
(31.15-80.25)\end{array}$ & $\begin{array}{l}54.09 \\
(31.63-88.56)\end{array}$ & $\begin{array}{l}43.99 \\
(28.12-71.62)\end{array}$ & $\begin{array}{l}40.71 \\
(30.54-92.13)\end{array}$ & 0.489 \\
\hline
\end{tabular}

Data are presented as the median value (25th-75th percentile). The differences in variable distributions among the groups before laparoscopic sleeve gastrectomy (LSG) were assayed with the Kruskal-Wallis test for independent samples (non-normally distributed variables). The differences in variable distributions within each group before versus after LSG were assayed with Wilcoxon signed-ranks for paired samples (non-normally distributed variables). ${ }^{*} p<0.05$; $* * p<0.01$; *** $p<0.001$. M, male; F, female; BMI, body mass index; FPG, fasting plasma glucose; HOMA-IR, homeostasis model assessment-insulin resistance index; AST, aspartate aminotransferase; ALT, alanine aminotransferase; GGT, gamma-glutamyl transferase; PLTS, platelets; T Chol, total cholesterol; LDL, low-density lipoprotein cholesterol; HDL, high-density lipoprotein cholesterol; N-HDL, non-HDL-cholesterol; TG, triglycerides; hs-CRP, high-sensitivity C-reactive protein; IL-6, interleukin-6; SCCA-IgM, squamous cell carcinoma antigen - immunoglobulin M complexes.

\section{Results}

\section{Study of Patients with Obesity Divided into Classes of Metabolic Risk}

The anthropometric characteristics and biochemical parameters before and after LSG are presented in Table 1, where patients with severe obesity have been divided on the basis of liver steatosis assessed by US in the no steatosis (16 patients) and steatosis (40 patients) subgroups. Patients with steatosis had statistically significant higher levels of ALT and ALT/ AST ratio than patients without steatosis (Table 1, column " $p$ before"). Insulin levels and the HOMA-IR index were higher in the subgroup with steatosis even if they were not significant, 


\begin{tabular}{l|l}
\hline DOI: 10.1159/000499717 & $\begin{array}{l}\text { (c) 2019 The Author(s). Published by S. Karger AG, Basel } \\
\text { www.karger.com/ofa }\end{array}$ \\
\hline
\end{tabular}

Bettini et al.: SCCA-IgM in NAFLD, Obesity, Prediabetes, and Diabetes

Table 3. Characteristics of the 4 case report patients

\begin{tabular}{|c|c|c|c|c|c|c|c|c|}
\hline $\begin{array}{l}\text { Patient } \\
\text { No. }\end{array}$ & Symbols & Glycaemic profile & $\begin{array}{l}\text { Dyslipid- } \\
\text { aemia }\end{array}$ & $\begin{array}{l}\text { Trans- } \\
\text { aminases }\end{array}$ & $\begin{array}{l}\text { Inflammation } \\
\text { markers }\end{array}$ & Thyroid disease & Steatosis & Other \\
\hline 1 & $\mathbf{\square}$ & ob preDM & No & $\mathrm{N}$ & $\begin{array}{l}\uparrow \\
\text { (hs-CRP) }\end{array}$ & A hypothyroidism & Yes by US & $\mathrm{AR}$ \\
\hline 2 & $\square$ & ob T2DM ID & Yes & $\mathrm{N}$ & $\begin{array}{l}\uparrow \\
\text { (hs-CRP) }\end{array}$ & A hyperthyroidism TC & Yes by US & \\
\hline 3 & $\boldsymbol{\Delta}$ & ob N with IR & Yes & $\uparrow$ & $\begin{array}{l}\uparrow \\
\text { (IL-6, hs-CRP) }\end{array}$ & NA hypothyroidism, LT & Yes by US & OSAS \\
\hline 4 & $\triangle$ & ob T2DM ID & Yes & $\uparrow$ & $\begin{array}{l}\uparrow \\
\text { (IL-6, hs-CRP) }\end{array}$ & NA hypothyroidism & $\begin{array}{l}\text { Yes by US } \\
\text { and NASH by } \\
\text { biopsy }\end{array}$ & \\
\hline
\end{tabular}

The symbols are used in Figure 2a and Figure 5a. ob N, patients with obesity and normoglycaemia; ob preDM, patients with obesity and prediabetes; ob T2DM, patients with obesity and diabetes; IR, insulin resistance; ID, insulin dependence; N, normal values; hs-CRP, high-sensitivity C-reactive protein; IL-6, interleukin-6; A, autoimmune; NA, non-autoimmune; LT, levothyroxine replacement therapy; TC, thiamazole-controlled; US, ultrasound scan; NASH, nonalcoholic steatohepatitis; AR, rheumatoid arthritis; OSAS, obstructive sleep apnea syndrome; $\uparrow$, above the normal value.

Fig. 1. Frequency of steatosis in patients with obesity and different glycaemic profiles. The presence of steatosis (frequency percentage) was evaluated by US in patients with obesity and normoglycaemia (ob N, white bars), in patients with obesity and prediabetes (ob preDM, grey bars), and in patients with obesity and diabetes (ob T2DM, black bars). Statistical analysis was performed by $\chi^{2}$ test.

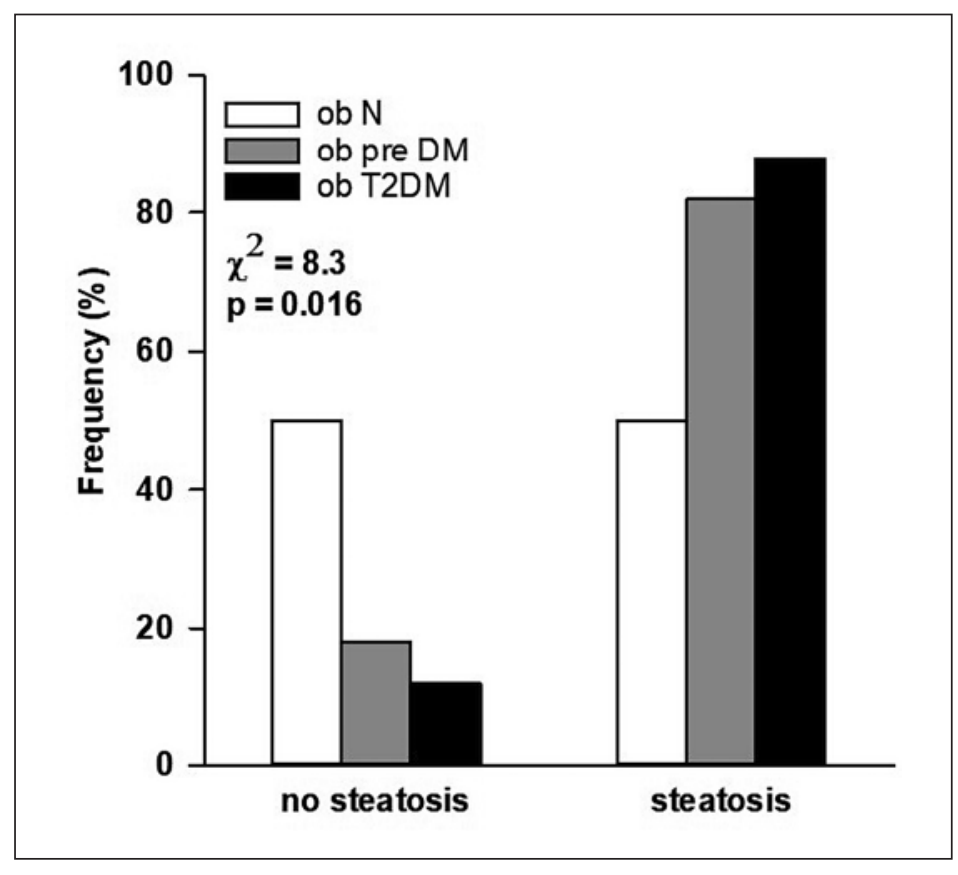

probably because of the interindividual variability. After LSG, we observed a significant decrease of most analysed parameters and, particularly, the reduction in AST, ALT, ALT/AST ratio, and GGT levels after LSG intervention achieved a higher significance level in patients with rather than without steatosis.

The patients were further analysed according to glycaemic profile and divided into 3 subgroups: patients with obesity and normoglycemia (ob N), patients with obesity and prediabetes (ob preDM), and patients with obesity and diabetes (ob T2DM), on the basis of ADA 


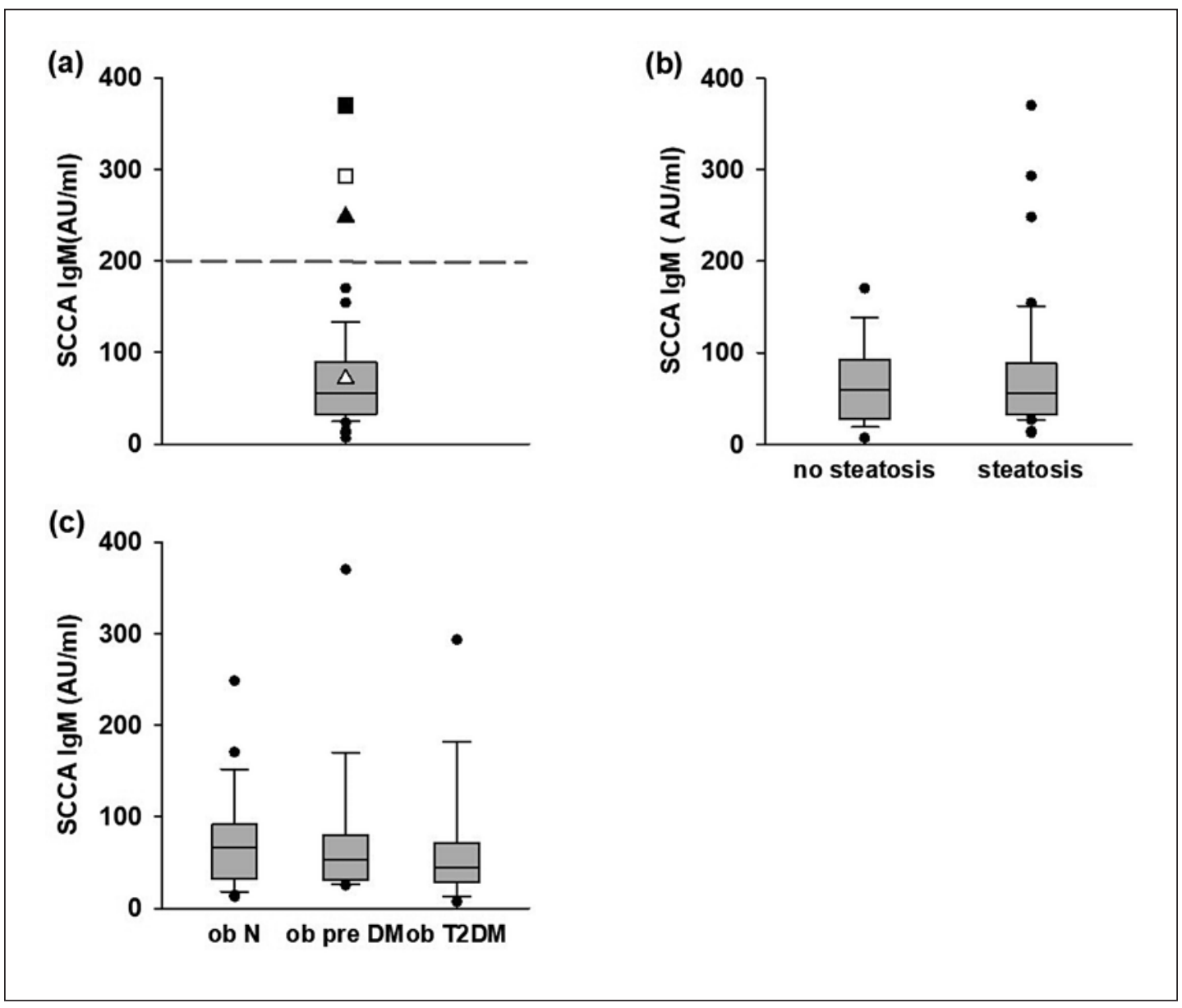

Fig. 2. Distribution of SCCA-IgM values in patients with obesity. a Distribution of SCCA-IgM values in 56 patients with obesity; the cut-off value is indicated by the dashed line. Patient 1 (black square), patient 2 (white square), and patient 3 (black triangle) had an SCCA-IgM value above the upper normal limit. Patient 4 (white triangle), who had a biopsy-proven diagnosis of NASH, had normal SCCA-IgM values. The clinical features of patients 1-4 are reported in Table 3. b Distribution of SCCA-IgM in patients with obesity with or without liver steatosis assessed by US. Statistical analysis was performed by independent samples Mann-Whitney U test ( $p=0.849$ ). c Distribution of SCCA-IgM values in patients with obesity and normoglycemia (ob N, $n=22$ ), patients with obesity and prediabetes (ob preDM, $n=17$ ), and patients with obesity and diabetes (ob T2DM, $n=$ 17). Statistical analysis was performed by independent samples Kruskal-Wallis test ( $p=0.489)$. Results are reported as box plot graphs: the box represents the lower and upper quartiles, the line in the box represents the median, the whiskers show the lowest and highest values, and the outliers are represented by black circles.

criteria [35] (Table 2). Twenty-two patients were normoglycaemic, 17 were prediabetic (impaired fasting glycaemia or impaired glucose tolerance at the OGTT), and 17 were diabetic, 4 of whom were already on an insulin regimen. The 3 subgroups were statistically different for levels of FPG, HOMA-IR index, and TG (Tables 2, column " $p$ before"). All subgroups of patients with obesity obtained a relevant weight loss together with a significant improvement after LSG of lipid profile, transaminases, and inflammation markers. LDL after LSG was significantly ameliorated in ob $\mathrm{N}$ and ob preDM in respect to the ob T2DM subgroup, probably because ob T2DM assumed statin therapy already before surgery.

We observed that the presence of steatosis before bariatric surgery, assessed by US examination, was associated with a worsening glycaemic profile (ob $\mathrm{N}$ vs. ob preDM vs. ob 


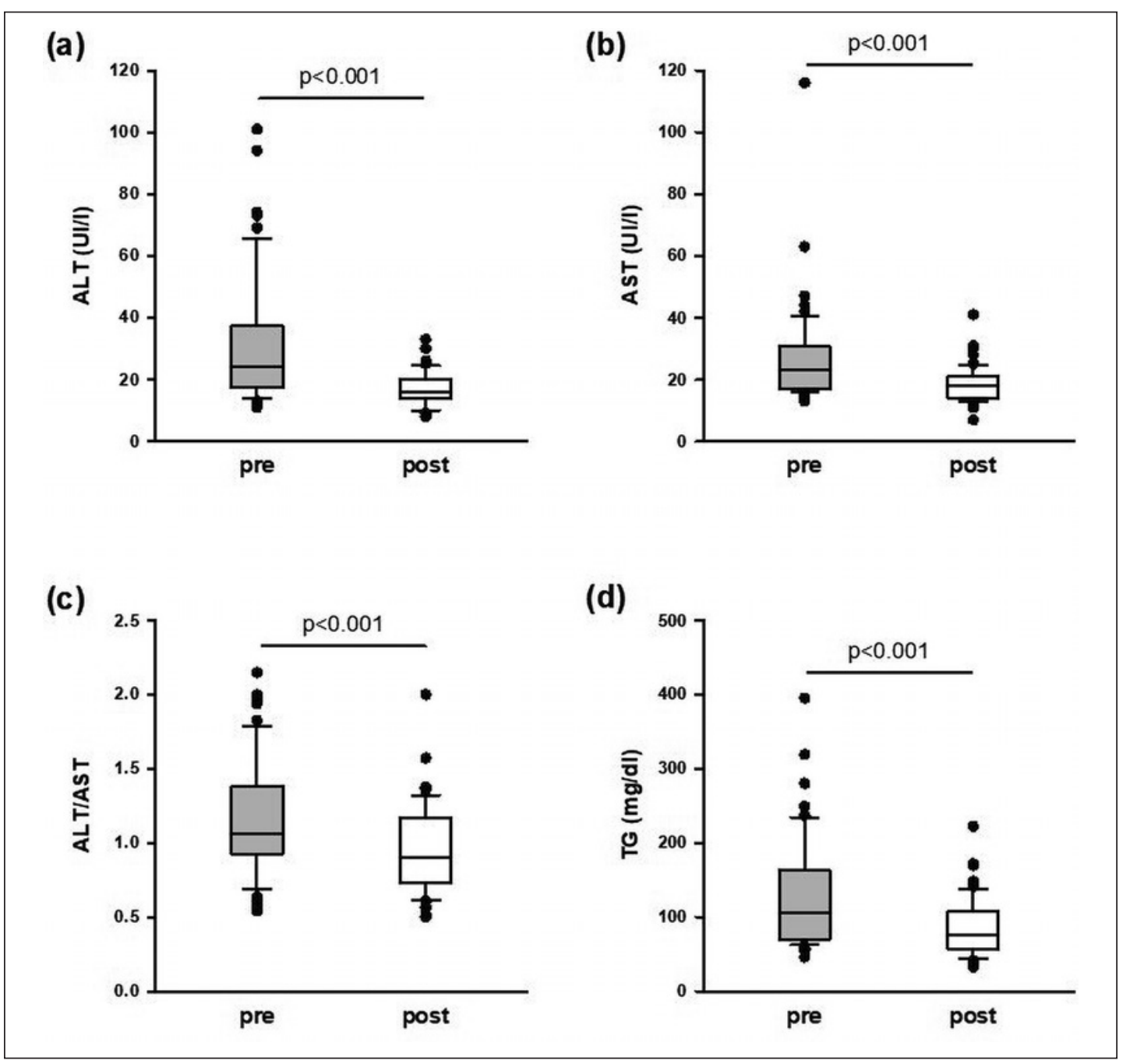

Fig. 3. Effects of LSG on the liver in patients with obesity. ALT (a), AST (b), ALT/AST ratio (c), and TG (d) values were determined in patients with obesity $(n=56)$ before (pre, grey boxes) and after (post, white boxes) surgery. Results are reported as box plot graphs with the lowest and highest values (whiskers), medians (lines), and outliers (black circles). Statistical analysis was performed by the Wilcoxon signed-rank test.

T2DM, $\chi^{2}$ test $p=0.016$ ) as shown in Figure 1. In fact, while $50 \%$ of ob $\mathrm{N}$ displayed steatosis and $50 \%$ were without steatosis (no steatosis), the majority of patients with obesity and early glycaemic impairment (ob preDM) also presented liver steatosis. Interestingly, a high frequency of steatosis $(82 \%)$ was already present in patients with obesity and prediabetes, similarly to the ob T2DM subgroup (88\%).

In these patients with severe obesity divided into subgroups as previously described, we quantified SCCA-IgM as a marker of liver damage. Only 3 patients had a SCCA-IgM value above the normal limit (cut-off value $200 \mathrm{AU} / \mathrm{mL}$ ) [33] (Fig. 2a). The clinical features of the 3 patients with increased SCCA-IgM are presented in Table 3. We observed that all patients had a thyroid disease and 2 of them presented an autoimmune disorder. Furthermore, it is interesting to note that a patient with a liver biopsy positive for NASH displayed normal levels of SCCA-IgM (patient 4 in Table 3 and represented by a white triangle in Fig. 2a). Figure $2 \mathrm{~b}$ shows the plotted titre of SCCA-IgM in patients with and without steatosis $(p=0.849)$. All 3 SCCA-IgM- 
(a)

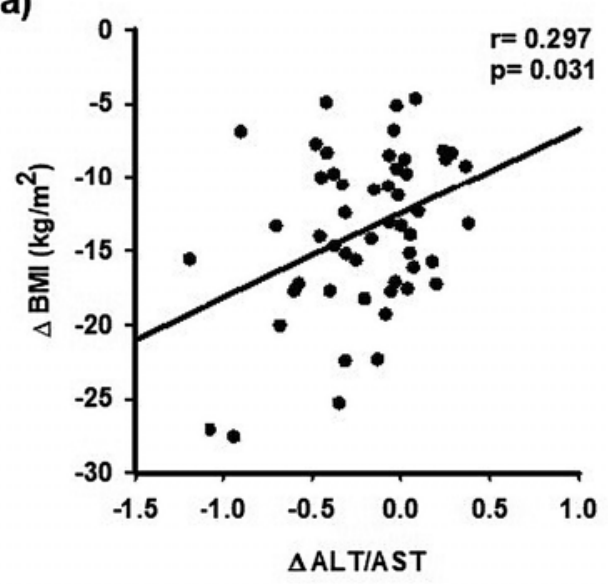

(c)

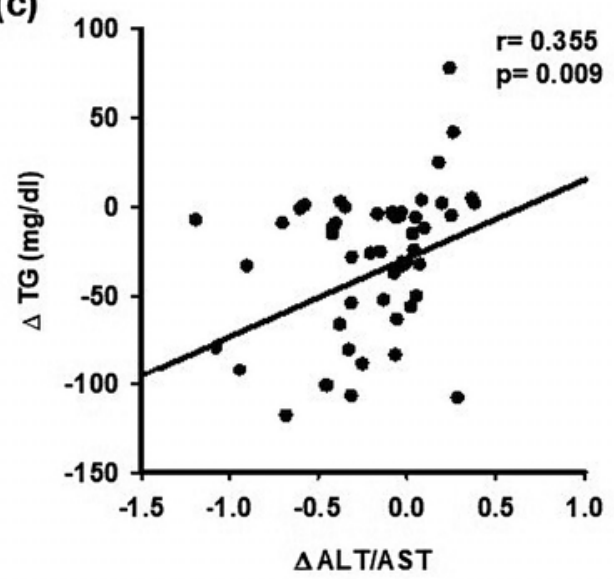

(b)

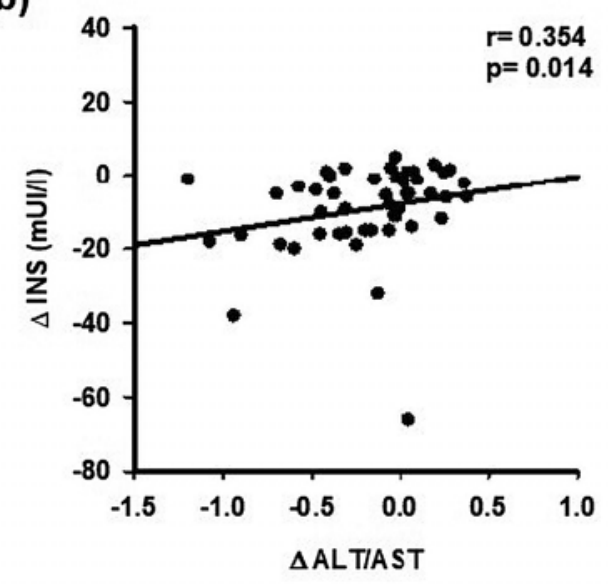

(d)

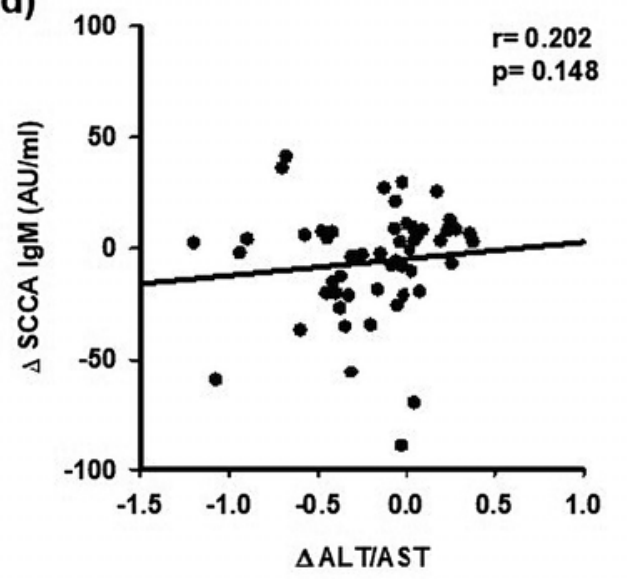

Fig. 4. Correlation analysis between the differences $(\Delta)$ before and after LSG in patients with obesity. $\Delta$ ALT/ AST ratio was correlated with differences in BMI (a), insulin (INS; b), TG (c), and SCCA-IgM (d) plasma levels in all patients with obesity $(n=56)$. Statistical analysis was performed by Spearman's correlation.

positive patients had steatosis. We did not observe any difference in SCCA-IgM titres among ob N, ob preDM, and ob T2DM ( $p=0.489$; Fig. $2 c$ ). Before bariatric surgery, we did not observe any correlation between SCCA-IgM and all analysed variables (serum lipids, ALT, AST, ALT/ AST, GGT, FPG, insulin, HOMA-IR, platelets count, hs-CRP, IL-6, leptin).

\section{Study of Anthropometric and Biochemical Parameters before and after LSG}

After surgery, overall weight loss was $20.4 \pm 9.5 \%$, with relative reductions in ALT ( $p<$ 0.001 ; Fig. 3a), AST ( $p<0.001$; Fig. 3b), ALT/AST ratio ( $p<0.001$; Fig. 3c), glucose $(p<0.001)$, insulin ( $p<0.001)$, platelets $(p<0.001)$, and serum lipids, in particular TG $(p<0.001$; Fig. 3d), hsCRP $(p<0.001)$, IL-6 $(p<0.05)$, and leptin $(p<0.001)$. The ALT/AST ratio decrease after surgery correlated with BMI ( $r=0.297, p=0.031$; Fig. 4a), insulin levels $(r=0.354, p=0.014$; Fig. 4 b), and plasma TG reduction ( $r=0.355, p=0.009$; Fig. 4c). $\Delta$ ALT $/$ AST ratio also presented a positive correlation with the decrease in the HOMA index $(r=0.280, p=0.050$; data not shown). Finally, $\triangle$ ALT/AST ratio was not correlated with the decrease of SCCA-IgM levels $(r=0.202, p=0.148$; Fig. 4 d). 


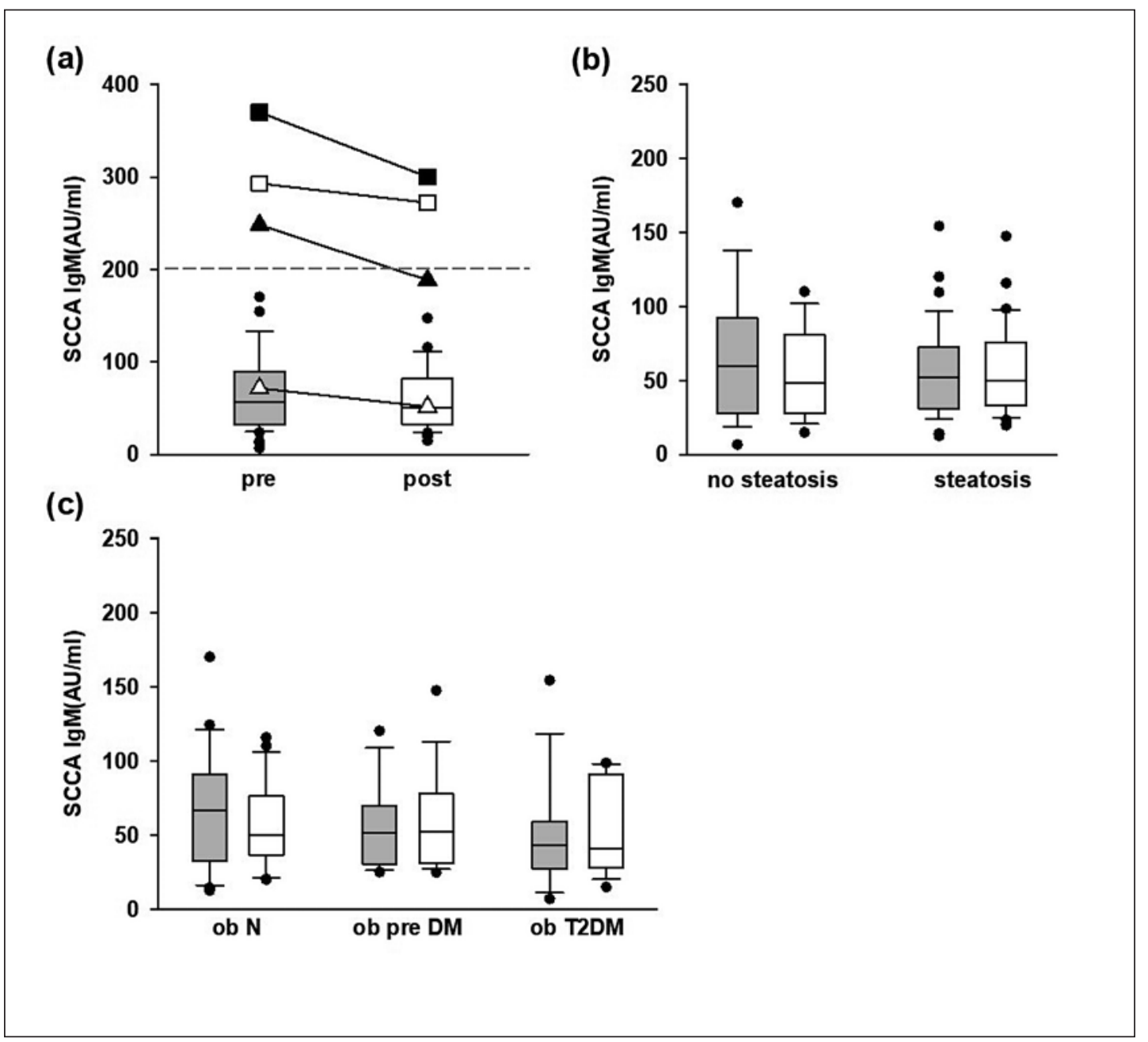

Fig. 5. Distribution of SCCA-IgM levels before (pre, grey boxes) and after (post, white boxes) LSG in patients with obesity. a Distribution of SCCA-IgM values in 56 patients with obesity pre/post LSG. Patient 1 (black square), patient 2 (white square), patient 3 (black triangle), and patient 4 (white triangle) were the 4 case report patients described in Table 3. Statistical analysis was performed by the Wilcoxon signed-rank test $(p=0.269)$. b Distribution of SCCA-IgM in patients with obesity displaying liver steatosis $(n=40)$ or not ( $n=16)$ assessed by US. c Distribution of SCCA-IgM values in patients with obesity and normoglycemia (ob N, $n=22$ ), patients with obesity and prediabetes (ob preDM, $n=17$ ), and patients with obesity and diabetes (ob T2DM, $n=17$ ). Statistical analysis was performed by the Wilcoxon signed-rank test for coupled data and with Mann-Whitney U test for independent samples (b) and with Kruskal-Wallis test for independent samples (c). No statistically significant differences were found: no steatosis (pre vs. post) $p=0.323$; steatosis (pre vs. post) $p=0.550$; no steatosis versus steatosis, pre $p=0.849$, post $\mathrm{p}=0.556$ (b); in ob N (pre vs. post) $p=0.051$, in ob preDM (pre vs. post) $p=0.927$, in ob T2DM (pre vs. post) $p=0.758$; ob N versus ob preDM versus ob T2DM, pre $p=0.489$, post $p=0.870(\mathbf{c})$. Results are presented as box plot graphs with the lowest and highest values (whiskers), the medians (lines), and outliers (black circles).

The decrease of SCCA-IgM levels was not significant a year after bariatric surgery $(p=0.267)$, even if we found a trend of reduction in the 3 SCCA-IgM-positive patients (above the cut-off line), as represented in Figure 5a. Moreover, we did not find any association between the decrease of SCCA-IgM titres and variations of body weight, HOMA index, platelets, ALT, AST, GGT, FPG level, or inflammation markers when considering all patients. SCCA-IgM levels did not present statisti- 
Bettini et al.: SCCA-IgM in NAFLD, Obesity, Prediabetes, and Diabetes

cally significant changes in patients with steatosis before and after LSG (pre [grey bars] vs. post [white bars], $p=0.550$ ), or in patients without steatosis (pre vs. post, $p=0.323$ ), or considering the two groups together (steatosis vs. no steatosis: post, $p=0.556$; Fig. 5b). The decrease of SCCA-IgM levels after LSG in each group according to glycaemic profile (ob N, ob preDM, ob T2DM) did not show significant differences: in ob $\mathrm{N}$ (pre vs. post) $p=0.051$, in ob preDM $p=$ 0.927 , and in ob T2DM $p=0.758$. Moreover, the decrease of SCCA-IgM considering the three groups (ob N vs. ob preDM vs. ob T2DM) was not statistically significant (post $p=0.870$; Fig. $5 \mathrm{c}$ ).

\section{Discussion}

Given the high prevalence of NAFLD, liver biopsy cannot be proposed to all patients with severe obesity who are candidate to bariatric surgery. As a consequence, a non-invasive diagnosis of NAFLD and its spectrum of conditions still remains a key challenge.

The clinical usefulness of monitoring SCCA-IgM immune-complexes in chronic liver disease has been evaluated in several studies [19-22]. SCCA-IgM is a known biomarker of progression to hepatocellular carcinoma from viral hepatitis, but its role in the diagnosis and progression of NAFLD has not yet been investigated.

Beale et al. [36] first studied SCCA-1 and other proteins as surveillance biomarkers for hepatocellular cancer in non-alcoholic and alcoholic fatty liver disease: they concluded that SCCA-1 had no HCC surveillance benefit in steatohepatitis-related cirrhosis. However, this study was biased by an analytical issue as SCCA-1 was later found to have poor stability in serum and thus to be an unreliable biomarker. Better analytic results were indeed obtained by testing IgM-linked SCCA instead of free SCCA protein [19], and isoform-specific immunoenzymatic assays have been set up using commercially available monoclonal antibodies.

In the literature there are not any studies on the behaviour of circulating SCCA-IgM in patients with metabolic liver disease and no studies of patients with obesity undergoing bariatric surgery. The aim of our study was to assess a possible role of SCCA-IgM in identifying patients with NAFLD and patients with a worse prognosis, speculating that SCCA-IgM could be related to the metabolic liver disease. The multiple-hit hypothesis, including diet, environmental and genetic factors, together with obesity per se and low-grade inflammation, describes the pathogenesis of NAFLD and the risk of progression to inflammation and fibrosis (NASH) or persistence in a stable stage of disease (NAFLD) [37, 38].

In order to study a potential role of SCCA-IgM in NAFLD we looked for any correlation with this biomarker and the presence of steatosis, anthropometric (weight, BMI) and metabolic (lipidic and glycaemic profile) parameters, levels of liver enzymes (ALT, AST, GGT), ALT/AST ratio, and inflammation markers (hs-CRP, IL-6, platelets count). Platelet count has been shown to be a valuable surrogate biomarker predicting the severity of fibrosis in NAFLD patients [39] and could be used to predict the activity of the disease [40].

We divided patients according different classes of risk of metabolic liver disease. Considering the glycaemic profile, we did not find increased values of SCCA-IgM in ob preDM and ob T2DM with respect to ob N. In the same way, patients with steatosis did not have levels of SCCA-IgM higher than those of patients without steatosis, even if the 3 patients with SCCA-IgM titres above the normal values also displayed liver steatosis.

Interestingly, in our population of patients with obesity we described a tight association between prediabetes and T2DM and the presence of steatosis. Many studies have confirmed a very high prevalence of NAFLD in individuals with T2DM [3, 4], but we firstly showed that patients with prediabetes already have a similar percentage of steatosis as patients with T2DM. Thus, we could speculate that prediabetes also represents a risk factor for NAFLD to the same extent as T2DM in patients with obesity. Nevertheless, we emphasise that metabolic 
risk factors do not represent the only predictive condition of NAFLD, as we can see in our population with a proportion of $50 \%$ of ob $\mathrm{N}$ with steatosis $[6,7]$.

Bariatric surgery is currently regarded as the only treatment option that provides sustained and successful long-term weight loss and improvement of obesity-related comorbidities [41]. The impact of bariatric surgery on the course of NAFLD in individuals with obesity has been extensively studied, and in the most recent IFSO consensus statement it is stated that bariatric surgery leads to the reversal or significant improvement of NAFLD and NASH [42]. In our patients with obesity, we observed a significant decrease of all biochemical and metabolic parameters assayed after bariatric surgery, clearly showing that LSG was effective in improving serum cytokine and the adipocytokine profile, thus ameliorating systemic inflammatory and metabolic status $[43,44]$. We noted a statistically significant reduction of platelet count, as Johansson et al. [45] also showed in 124 morbidly obese nondiabetic patients 12 months after Roux-en Y gastric bypass. In our population this decrease was not evident in ob T2DM, perhaps due to a more severe inflammation state and a higher degree of fibrosis than the ob $\mathrm{N}$ and ob preDM subgroups, which also persisted after surgery. Weight loss after LSG resulted in a significant reduction of ALT, AST, GGT, and ALT/AST ratio, and we proved that the improvement of transaminases is related to the degree of weight loss. This fact has already been described in other recent studies [23-26]. In the Swedish Obese Subjects Study [46] the reduction in ALT levels was proportional to the degree of weight loss. Similarly, the prevalence of ALT/AST ratio $<1$ was lower in the surgery compared to the behavioural group at both the 2- and 10-year follow-up. We observed a decreased trend of SCCA-IgM after LSG, even if not statistically significant. Moreover, the 3 patients with SCCA-IgM values above the normal limit, and the patient with a biopsy-proven NASH, also showed a reduction of serpin complex after LSG. No difference in SCCA-IgM levels has been reported 12 months after LSG when dividing patients into groups according to the presence of steatosis or considering metabolic comorbidities as prediabetes and T2DM. It has been demonstrated that in patients with chronic hepatitis undergoing standard antiviral treatment and achieving a sustained virological response there was a significant decrease in serum levels of SCCA-IgM at the end of treatment and up to 1 year of follow-up [21]. We might speculate that the damage inferred by a metabolic imbalance could be different from that caused by viral chronic infection, in which the use of SCCA-IgM has been validated [14,16, 19-22]. Indeed, it is well known that adipose tissue is characterized by a state of low-grade inflammation that is the basis of the pathogenesis of NAFLD [38]. In this context, we can hypothesize that SCCA-IgM may not be a sufficient inflammation marker and it could explain the absence of association between the serpin complex and inflammation factor levels (hs-CRP, IL-6, platelets) in patients with obesity before and after LSG. We suggest that the 3 positive patients might present a higher inflammation status (i.e., autoimmunity) than other patients with normal values of SCCA-IgM.

No robust data on the effects of LSG on liver fat are actually available and the exact mechanisms that lead to the improvement in NAFLD following bariatric surgery are not completely understood $[23,24,26,27]$. Our study proved that LSG have a significant positive effect on biochemical parameters of NAFLD with a correlation between the improvement of ALT/AST ratio and the decrease of BMI and TG. These data suggest that weight reduction has a positive effect on liver transaminases and chronic liver damage. Moreover, we observed a statistically significant correlation between ALT/AST ratio, insulin levels, and IR expressed by the HOMA index. This fact is consistent with the literature $[38,46,47]$ in proving the connection between obesity, IR, steatosis, and liver injury. In a cross-sectional study [47], steatosis and fibrosis were associated with T2DM, both in terms of fasting glycaemia and HbA1c. These findings support the tight association between NAFLD and diabetes, and our study suggests that, considering the same percentage of steatosis displayed in ob preDM and ob T2DM, prediabetes may be a risk factor of NAFLD. Additionally, the fact that the decrease in TG is associated 
Bettini et al.: SCCA-IgM in NAFLD, Obesity, Prediabetes, and Diabetes

with the decrease in ALT/AST, which in turn is associated with a BMI reduction, is consistent with the pathophysiology of NAFLD [37]. On the contrary, SCCA-IgM did not seem to have any association with the improvement of the transaminases and of the ALT/AST ratio.

Our study has some limitations. First, we could not differentiate NASH from NAFL or stage the degree of fibrosis with a pathological scoring system because we were not allowed to carry out liver biopsies in patients. Although liver biopsy is currently considered the gold standard to assess and grade liver disease, the procedure is invasive and associated with specific costs and complications. Liver biopsy is also affected by sampling variability with the risk that the tissue specimen might not be representative of the amount of hepatic fibrosis in the whole liver due to the heterogeneity in its distribution $[9,48]$. For these reasons we established the presence of NAFLD with the use of US combined with transaminases levels, as is suggested by actual guidelines for clinical practise $[1,2]$.

In conclusion, our data suggest that LSG with the achievement of a sustained weight loss improves fibrosis, ameliorating liver transaminases and chronic liver damage. NAFLD risk in patients with obesity, determined by US and serum transaminase levels, is not only tightly linked to diabetes but also to prediabetes. Further prospective studies are needed to detect the precise link between prediabetes and NAFLD in order to improve the screening and the management of patients with NAFLD, and to evaluate the timing to treat patients with prediabetes. SCCA-IgM is a biomarker of the progression to HCC from NASH and advanced fibrosis of viral origin, and we have highlighted the lack of any correlation between SCCA-IgM and the severity of fatty liver. Thus, our results suggest that levels of SCCA-IgM are not useful to identify patients with NAFLD or a worse prognosis. The identification of the mechanisms underlying the improvement process of liver disease after bariatric surgery could help to identify a validated and non-invasive biomarker or score, with the aim of saving a number of diagnostic liver biopsies.

\section{Statement of Ethics}

Informed consent was obtained from all individual participants included in the study. All procedures performed in studies involving human participants were in accordance with the ethical standards of the institutional and/or national research committee, and with the 1964 Helsinki declaration and its later amendments, or comparable ethical standards.

\section{Disclosure Statement}

Dr. Serra reports receiving personal fees from Pizeta Pharma and Astra Zeneca for his services as a speaker and scientific advisor. Dr. Busetto reports receiving personal fees from IntraPace, Pizeta Pharma, and Novo Nordisk for his services as a scientific advisor. Dr. Fassina is an employee of Xeptagen S.p.A. Dr. Vettor declares that he has been a consultant/advisor as well as having received grants/research support from Sanofi-Aventis. In addition, he has completed sponsored lectures for Novo Nordisk, Sanofi-Aventis, and AstraZeneca. All other authors declare no conflicts of interest.

\section{Funding Sources}

The study was conducted with institutional grants from the Centre for the Study and Integrated Treatment of Obesity, University of Padua, and financial support from Xeptagen. 
Bettini et al.: SCCA-IgM in NAFLD, Obesity, Prediabetes, and Diabetes

\section{References}

1 European Association for the Study of the Liver (EASL)European Association for the Study of Diabetes (EASD) European Association for the Study of Obesity (EASO). EASL-EASD-EASO Clinical Practice Guidelines for the management of non-alcoholic fatty liver disease. J Hepatol. 2016 Jun;64(6):1388-402.

2 Chalasani N, Younossi Z, Lavine JE, Charlton M, Cusi K, Rinella M, et al. The diagnosis and management of nonalcoholic fatty liver disease: Practice guidance from the American Association for the Study of Liver Diseases. Hepatology. 2018 Jan;67(1):328-57.

3 Bhala N, Younes R, Bugianesi E. Epidemiology and natural history of patients with NAFLD. Curr Pharm Des. 2013;19(29):5169-76.

4 Bugianesi E, McCullough AJ, Marchesini G. Insulin resistance: a metabolic pathway to chronic liver disease. Hepatology. 2005 Nov; 42(5):987-1000.

5 de Alwis NM, Day CP. Non-alcoholic fatty liver disease: the mist gradually clears. J Hepatol. 2008;48 Suppl 1:S104-12.

6 Subichin M, Clanton J, Makuszewski M, Bohon A, Zografakis JG, Dan A. Liver disease in the morbidly obese: a review of 1000 consecutive patients undergoing weight loss surgery. Surg Obes Relat Dis. 2015 Jan-Feb;11(1):137-41.

7 Hamaguchi M, Kojima T, Takeda N, Nakagawa T, Taniguchi H, Fujii K, et al. The metabolic syndrome as a predictor of nonalcoholic fatty liver disease. Ann Intern Med. 2005 Nov;143(10):722-8.

8 European Association for Study of LiverAsociacion Latinoamericana para el Estudio del Higado. EASL-ALEH Clinical Practice Guidelines: non-invasive tests for evaluation of liver disease severity and prognosis. J Hepatol. 2015 Jul;63(1):237-64.

9 Tapper EB, Lok AS. Use of liver imaging and biopsy in clinical practice. N Engl J Med. 2017 Aug;377(8):756-68.

10 Cleveland E, Bandy A, VanWagner LB. Diagnostic challenges of nonalcoholic fatty liver disease/nonalcoholic steatohepatitis. Clin Liver Dis (Hoboken). 2018 Apr;11(4):98-104.

11 Angulo P, Keach JC, Batts KP, Lindor KD. Independent predictors of liver fibrosis in patients with nonalcoholic steatohepatitis. Hepatology. 1999 Dec;30(6):1356-62.

12 Nanji AA, French SW, Freeman JB. Serum alanine aminotransferase to aspartate aminotransferase ratio and degree of fatty liver in morbidly obese patients. Enzyme. 1986;36(4):266-9.

13 Suminami Y, Kishi F, Sekiguchi K, Kato H. Squamous cell carcinoma antigen is a new member of the serine protease inhibitors. Biochem Biophys Res Commun. 1991 Nov;181(1):51-8.

14 Turato C, Buendia MA, Fabre M, Redon MJ, Branchereau S, Quarta S, et al. Over-expression of SERPINB3 in hepatoblastoma: a possible insight into the genesis of this tumour? Eur J Cancer. 2012 May;48(8):1219-26.

15 Chechlinska M, Kowalewska M, Brzoska-Wojtowicz E, Radziszewski J, Ptaszynski K, Rys J, et al. Squamous cell carcinoma antigen 1 and 2 expression in cultured normal peripheral blood mononuclear cells and in vulvar squamous cell carcinoma. Tumour Biol. 2010 Dec;31(6):559-67.

16 Turato C, Calabrese F, Biasiolo A, Quarta S, Ruvoletto M, Tono N, et al. SERPINB3 modulates TGF-beta expression in chronic liver disease. Lab Invest. 2010 Jul;90(7):1016-23.

17 Lunardi F, Villano G, Perissinotto E, Agostini C, Rea F, Gnoato M, et al. Overexpression of SERPIN B3 promotes epithelial proliferation and lung fibrosis in mice. Lab Invest. 2011 Jun;91(6):945-54.

18 Suminami Y, Nagashima S, Vujanovic NL, Hirabayashi K, Kato H, Whiteside TL. Inhibition of apoptosis in human tumour cells by the tumour-associated serpin, SCC antigen-1. Br J Cancer. 2000 Feb;82(4):981-9.

19 Beneduce L, Castaldi F, Marino M, Quarta S, Ruvoletto M, Benvegnù L, et al. Squamous cell carcinoma antigenimmunoglobulin M complexes as novel biomarkers for hepatocellular carcinoma. Cancer. 2005 Jun;103(12): 2558-65.

20 Biasiolo A, Chemello L, Quarta S, Cavalletto L, Bortolotti F, Caberlotto C, et al. Monitoring SCCA-IgM complexes in serum predicts liver disease progression in patients with chronic hepatitis. J Viral Hepat. 2008 Apr;15(4): 246-9.

21 Martini A, Fattovich G, Guido M, Bugianesi E, Biasiolo A, Ieluzzi D, et al. HCV genotype 3 and squamous cell carcinoma antigen (SCCA)-IgM are independently associated with histological features of NASH in HCVinfected patients. J Viral Hepat. 2015 Oct;22(10):800-8.

22 Pontisso P, Quarta S, Caberlotto C, Beneduce L, Marino M, Bernardinello E, et al. Progressive increase of SCCA-IgM immune complexes in cirrhotic patients is associated with development of hepatocellular carcinoma. Int J Cancer. 2006 Aug;119(4):735-40.

23 Lassailly G, Caiazzo R, Buob D, Pigeyre M, Verkindt H, Labreuche J, et al. Bariatric surgery reduces features of non-alcoholic steatohepatitis in morbidly obese patients. Gastroenterology. 2015 Aug;149(2):379-88.

24 Nickel F, Tapking C, Benner L, Sollors J, Billeter AT, Kenngott HG, et al. Bariatric Surgery as an Efficient Treatment for Non-Alcoholic Fatty Liver Disease in a Prospective Study with 1-Year Follow-up : BariScan Study. Obes Surg. 2018 May;28(5):1342-50.

25 Caiazzo R, Lassailly G, Leteurtre E, Baud G, Verkindt H, Raverdy V, et al. Roux-en-Y gastric bypass versus adjustable gastric banding to reduce nonalcoholic fatty liver disease: a 5-year controlled longitudinal study. Ann Surg. 2014 Nov;260(5):893-8.

26 Kalinowski P, Paluszkiewicz R, Ziarkiewicz-Wróblewska B, Wróblewski T, Remiszewski P, Grodzicki M, et al. Liver Function in Patients With Nonalcoholic Fatty Liver Disease Randomized to Roux-en-Y Gastric Bypass Versus Sleeve Gastrectomy: A Secondary Analysis of a Randomized Clinical Trial. Ann Surg. 2017 Nov;266(5): 738-45. 
Bettini et al.: SCCA-IgM in NAFLD, Obesity, Prediabetes, and Diabetes

27 Algooneh A, Almazeedi S, Al-Sabah S, Ahmed M, Othman F. Non-alcoholic fatty liver disease resolution following sleeve gastrectomy. Surg Endosc. 2016 May;30(5):1983-7.

28 Yumuk V, Tsigos C, Fried M, Schindler K, Busetto L, Micic D, et al.; Obesity Management Task Force of the European Association for the Study of Obesity. European Guidelines for Obesity Management in Adults. Obes Facts. 2015;8(6):402-24.

29 Bernante P, Foletto M, Busetto L, Pomerri F, Pesenti FF, Pelizzo MR, et al. Feasibility of laparoscopic sleeve gastrectomy as a revision procedure for prior laparoscopic gastric banding. Obes Surg. 2006 Oct;16(10): 1327-30.

30 Khov N, Sharma A, Riley TR. Bedside ultrasound in the diagnosis of nonalcoholic fatty liver disease. World J Gastroenterol. 2014 Jun;20(22):6821-5.

31 Siekmann L, Bonora R, Burtis CA, Ceriotti F, Clerc-Renaud P, Férard G, et al.; International Federation of Clinical Chemistry and Laboratory Medicine. IFCC primary reference procedures for the measurement of catalytic activity concentrations of enzymes at 37 degrees C. International Federation of Clinical Chemistry and Laboratory Medicine. Part 7. Certification of four reference materials for the determination of enzymatic activity of gamma-glutamyltransferase, lactate dehydrogenase, alanine aminotransferase and creatine kinase accord. Clin Chem Lab Med. 2002 Jul;40(7):739-45.

32 Friedewald WT, Levy RI, Fredrickson DS. Estimation of the concentration of low-density lipoprotein cholesterol in plasma, without use of the preparative ultracentrifuge. Clin Chem. 1972 Jun;18(6):499-502.

33 Buccione D, Fatti G, Gallotta A, Loggi E, Di Donato R, Testa L, et al. Serum SCCA-IgM as a predictor of hepatocellular carcinoma in patients with liver cirrhosis. Open J Gastroenterol. 2012;2(2):56-61.

34 Matthews DR, Hosker JP, Rudenski AS, Naylor BA, Treacher DF, Turner RC. Homeostasis model assessment: insulin resistance and beta-cell function from fasting plasma glucose and insulin concentrations in man. Diabetologia. 1985 Jul;28(7):412-9.

35 American Diabetes Association. Diagnosis and classification of diabetes mellitus. Diabetes Care. 2014 Jan;37 Suppl 1:S81-90.

36 Beale G, Chattopadhyay D, Gray J, Stewart S, Hudson M, Day C, et al. AFP, PIVKAII, GP3, SCCA-1 and follisatin as surveillance biomarkers for hepatocellular cancer in non-alcoholic and alcoholic fatty liver disease. BMC Cancer. 2008 Jul;8(1):200.

37 Buzzetti E, Pinzani M, Tsochatzis EA. The multiple-hit pathogenesis of non-alcoholic fatty liver disease (NAFLD). Metabolism. 2016 Aug;65(8):1038-48.

38 Polyzos SA, Kountouras J, Mantzoros CS. Adipose tissue, obesity and non-alcoholic fatty liver disease. Minerva Endocrinol. 2017 Jun;42(2):92-108.

39 Yoneda M, Fujii H, Sumida Y, Hyogo H, Itoh Y, Ono M, et al.; Japan Study Group of Nonalcoholic Fatty Liver Disease. Platelet count for predicting fibrosis in nonalcoholic fatty liver disease. J Gastroenterol. 2011 Nov; 46(11):1300-6.

40 Garjani A, Safaeiyan A, Khoshbaten M. Association between platelet count as a noninvasive marker and ultrasonographic grading in patients with nonalcoholic Fatty liver disease. Hepat Mon. 2015 Jan;15(1):e24449.

41 Sjöström L, Peltonen M, Jacobson P, Sjöström CD, Karason K, Wedel H, et al. Bariatric surgery and long-term cardiovascular events. JAMA. 2012 Jan;307(1):56-65.

42 De Luca M, Angrisani L, Himpens J, Busetto L, Scopinaro N, Weiner R, et al. Indications for Surgery for Obesity and Weight-Related Diseases: Position Statements from the International Federation for the Surgery of Obesity and Metabolic Disorders (IFSO). Obes Surg. 2016 Aug;26(8):1659-96.

43 Gumbau V, Bruna M, Canelles E, Guaita M, Mulas C, Basés C, et al. A prospective study on inflammatory parameters in obese patients after sleeve gastrectomy. Obes Surg. 2014 Jun;24(6):903-8.

44 Randell EW, Twells LK, Gregory DM, Lester KK, Daneshtalab N, Dillon C, et al. Pre-operative and post-operative changes in CRP and other biomarkers sensitive to inflammatory status in patients with severe obesity undergoing laparoscopic sleeve gastrectomy. Clin Biochem. 2018 Feb;52:13-9.

45 Johansson HE, Wåhlén A, Aldenbäck E, Haenni A. Platelet Counts and Liver Enzymes After Gastric Bypass Surgery. Obes Surg. 2018 Jun;28(6):1526-31.

46 Burza MA, Romeo S, Kotronen A, Svensson PA, Sjöholm K, Torgerson JS, et al. Long-term effect of bariatric surgery on liver enzymes in the Swedish Obese Subjects (SOS) study. PLoS One. 2013;8(3):e60495.

47 Cazzo E, Jimenez LS, Gestic MA, Utrini MP, Chaim FH, Chaim FD, et al. Type 2 Diabetes Mellitus and Simple Glucose Metabolism Parameters may Reliably Predict Nonalcoholic Fatty Liver Disease Features. Obes Surg. 2018 Jan;28(1):187-94.

48 Ratziu V, Charlotte F, Heurtier A, Gombert S, Giral P, Bruckert E, et al.; LIDO Study Group. Sampling variability of liver biopsy in nonalcoholic fatty liver disease. Gastroenterology. 2005 Jun;128(7):1898-906. 